\title{
Association of trial registration with the results and conclusions of published trials of new oncology drugs Nicolas Rasmussen*1, Kirby Lee ${ }^{2}$ and Lisa Bero²
}

Address: ${ }^{1}$ History \& Philosophy of Science, University of New South Wales, Sydney 2052, Australia and ${ }^{2}$ Department of Clinical Pharmacy, University of California, 3333 California St., San Francisco, CA 94118, USA

Email: Nicolas Rasmussen* - N.Rasmussen@unsw.edu.au; Kirby Lee - leek@pharmacy.ucsf.edu; Lisa Bero - berol@pharmacy.ucsf.edu

* Corresponding author

Published: 16 December 2009

Trials 2009, 10:116 doi:10.1186/1745-6215-10-116

This article is available from: http://www.trialsjournal.com/content/10/I/116

(C) 2009 Rasmussen et al; licensee BioMed Central Ltd.

This is an Open Access article distributed under the terms of the Creative Commons Attribution License (http://creativecommons.org/licenses/by/2.0), which permits unrestricted use, distribution, and reproduction in any medium, provided the original work is properly cited.

\begin{abstract}
Background: Registration of clinical trials has been introduced largely to reduce bias toward statistically significant results in the trial literature. Doubts remain about whether advance registration alone is an adequate measure to reduce selective publication, selective outcome reporting, and biased design. One of the first areas of medicine in which registration was widely adopted was oncology, although the bulk of registered oncology trials remain unpublished. The net influence of registration on the literature remains untested. This study compares the prevalence of favorable results and conclusions among published reports of registered and unregistered randomized controlled trials of new oncology drugs.
\end{abstract}

Methods: We conducted a cross-sectional study of published original research articles reporting clinical trials evaluating the efficacy of drugs newly approved for antimalignancy indications by the United States Food and Drug Administration (FDA) from 2000 through 2005. Drugs receiving first-time approval for indications in oncology were identified using the FDA web site and Thomson Centerwatch. Relevant trial reports were identified using PubMed and the Cochrane Library. Evidence of advance trial registration was obtained by a search of clinicaltrials.gov, WHO, ISRCTN, NCI-PDQ trial databases and corporate trial registries, as well as articles themselves. Data on blinding, results for primary outcomes, and author conclusions were extracted independently by two coders. Univariate and multivariate logistic regression identified associations between favorable results and conclusions and independent variables including advance registration, study design characteristics, and industry sponsorship.

Results: Of 137 original research reports from 115 distinct randomized trials assessing 25 newly approved drugs for treating cancer, the 54 publications describing data from trials registered prior to publication were as likely to report statistically significant efficacy results and reach conclusions favoring the test drug (for results, $\mathrm{OR}=1.77 ; 95 \% \mathrm{Cl}=0.87$ to $3.6 \mathrm{I}$ ) as reports of trials not registered in advance. In multivariate analysis, reports of prior registered trials were again as likely to favor the test drug $(O R=1.29 ; 95 \% \mathrm{Cl}=0.54$ to 3.08$)$; large sample sizes and surrogate outcome measures were statistically significant predictors of favorable efficacy results at $p<0.05$. Subgroup analysis of the main reports from each trial $(n=115)$ similarly indicated that registered trials were as likely to report results favoring the test drug as trials not registered in advance $(\mathrm{OR}=\mathrm{I} . \mathrm{II} ; 95 \% \mathrm{Cl}=0.44$ to 2.80$)$, and also that large trials and trials with nonstringent blinding were significantly more likely to report results favoring the test drug.

Conclusions: Trial registration alone, without a requirement for full reporting of research results, does not appear to reduce a bias toward results and conclusions favoring new drugs in the clinical trials literature. Our findings support the inclusion of full results reporting in trial registers, as well as protocols to allow assessment of whether results have been completely reported. 


\section{Background}

The clinical trial literature is known to be a biased source of evidence on therapeutic efficacy. A number of potential mechanisms accounting for bias have been identified [1]. Selective publication of "statistically significant" results favoring new treatments is one well-established problem [2-7]. Intrinsic factors of trial design can also lead to bias in randomized controlled trials of drug efficacy [8,9]. Specific design features that have been associated with bias in reported trials include blinding $[8,10,11]$, sample size [9,11-13], and choice of comparator [14-17]. Particular data handling and analysis procedures have been associated with bias $[13,18,19]$ and also incomplete reporting of outcomes [2,20-22]. Trials and investigators supported by pharmaceutical companies are more likely to report results and conclusions favorable towards the sponsor's product compared to placebo $[13,14,23,24]$, or to the drugs of other manufacturers $[11,25]$, through mechanisms that remain incompletely characterized.

The public registration of clinical trials has been introduced, partly to satisfy ethical obligations of human experimentation [26-30], and partly to reduce bias via several mechanisms (increasing scrutiny of design, encouraging publication, and encouraging full outcome reporting) [7,31-33]. United States Federal law in 1997 mandated a national registry http://www.clinicaltrials.gov, which was operational by 1999 [30]. However the registry was at first little used except in a few fields, notably oncology (where it facilitated patient recruitment) [34]. Since the International Committee of Medical Journal Editors instituted a policy requiring prior trial registration for publication in cooperating journals from late 2005 [29], registration has become much more common and may soon be a universal regulatory requirement [30,35]. However, doubts remain about registration's adequacy in principle for preventing bias due to trial design $[36,37]$. Furthermore, there is evidence that registration alone may not greatly influence biased outcome reporting: among registered trials in cardiology, rheumatology, and gastroenterology that have been published, many showed discrepancies between primary outcomes as reported and outcomes as registered, the vast majority of which tended to favor statistically significant results [37]. Similarly, advance registration of trials may not greatly discourage selective publication: the majority of registered trials across all fields remain unpublished, and are less likely to be published if industry sponsored [38].

One of the main goals of trial registration is to reduce bias in the literature through some combination of effects on the mechanisms favoring new treatments. Oncology is one field in which published results and conclusions have been found to exhibit a bias favoring new drugs, associated statistically with drugmaker sponsorship and with particular trial design features [39]. A minority of oncology trials registered before September 2004 have reached publication, indicating that the possibility of publication bias in this field also remains [40]. In oncology, high quality randomized clinical trials (those of the National Cancer Institute's Cooperative Oncology Groups) have in the long run favored new treatments very nearly as often as comparator treatments, making this field unusually advantageous for studying predictors of favorable outcomes in that conditions approximately consistent with equipoise have been demonstrated [41]. To assess whether registration is associated with any net difference in the prevalence of results and conclusions favoring new drugs in the clinical trial literature, we conducted a crosssectional study of randomized clinical trial reports of oncology drugs approved by the United States Food and Drug Administration (FDA) from 2000 through 2005. We hypothesized that advance trial registration is associated with statistically non-significant efficacy outcomes in published trial reports, when controlling for other known predictors of reported results such as funding source, publication characteristics, and trial design characteristics.

\section{Methods \\ Search strategy}

We searched drug approvals by year on the FDA web site http://www.accessdata.fda.gov/scripts/cder/drugsatfda/

index.cfm as well as Thomson CenterWatch http:// www.centerwatch.com/drug-information/fda-approvals/. We identified 25 relevant drugs granted first-time FDA approval for oncology indications in the 2000-2005 period, including both new molecular entities and older drugs receiving new indications. We electronically searched PubMed and the Cochrane Library in mid-October 2007 to identify reports of randomized controlled trials including these drugs. Search terms were the generic name(s) of the drugs of interest, in PubMed limited to "randomized" or "randomised", "clinical trials", and "humans". Our search included articles published in any language. The search was updated in late February 2009 to expand the sample so as to include reports published through the end of 2008. Thus trial reports on all drugs of interest were collected for a full nine-year interval surrounding FDA approval, except for those drugs approved in 2005, for which only eight years of literature was searched.

\section{Published Articles}

We reviewed abstracts of all citations and retrieved articles based on the following inclusion criteria: (1) prospective randomized controlled trial; (2) oncology drug of interest compared to a treatment arm not including that drug (e.g., placebo or active control); (3) efficacy measured in human cancer patients; (4) efficacy measured in terms of direct effects on malignancy (not treatment-related 
pathology or supportive care only); (5) original research, defined as studies that appeared to present original data and did not specifically state that they were reviews. Studies with the primary objective of assessing the effect of the oncology drug of interest combined with another treatment were included, so long as one treatment arm including the drug of interest was compared with an otherwise identical arm lacking it, or with another treatment in its place. In the case of oxaliplatin, approved in 2002 only in combination with 5-fluorouracil and leucovorin, oxaliplatin was defined as the chief drug of interest and only trials comparing the approved combination to a treatment arm not containing oxaliplatin were included.

The following exclusion criteria were used to screen all abstracts: (1) studies not evaluating clinical efficacy outcomes (e.g., pharmacodynamic studies); (2) editorials, letters-to-the-editor, commentaries, abstracts, reviews, studies only describing a trial but not results; (3) studies presenting only an economic analysis; (4) retrospective analysis of outcome predictors not reporting any new efficacy data; (5) studies in which the oncology drug of interest was present in all arms of the trial; (6) studies published more than four years before or after the year of the oncology drug's approval in the 2000 to 2005 interval; (7) studies presenting pooled data from multiple trials, where individual trial results are not interpretable separately; (8) studies in which the primary efficacy outcome is not assessed statistically or not reported sufficiently for statistical assessment; and (9) strictly biological or in vitro analyses. We did not identify any exact duplicate publications. As we were interested in published trial reports, we did include multiple publications from the same underlying trial if the publications reported different data. Where any doubt about a publication's eligibility for inclusion remained after reading the abstract, it was provisionally included for a full reading.

\section{Data extraction}

One investigator (N.R.) who was not blinded to author names and affiliations, funding sources, and financial disclosure extracted all data from each article. All data were extracted from articles before a search of the registration status of the underlying trial was conducted. A second coder (K.L.) who was blinded to registration status (except where it was stated in the publication) independently extracted data on blinding, identification and results of primary outcomes, and author conclusions. Inter-rater agreement was very good to excellent, with Kappa values ranging from 0.85 to 0.95 for double-coded items. In cases of disagreement the two coders discussed the papers and reached agreement.

We extracted data on trial registration status and publication characteristics that have been shown to be independ- ently related to publication of favorable results or conclusions of drug studies, as follows [8-12,23].

\section{Registration status}

For all articles the registration status of the underlying trial was sought by searching cancer type and drug names in key databases. These included the U.S. National Institutes of Health trial registry http://www.clinicaltrials.gov, the International Standard Randomized Controlled Trial Number Register http://isrctn.org, the WHO International Clinical Trials Registry platform http://www.who.int/tri alsearch/, the U.S. National Cancer Institute PDQ Comprehensive Cancer database http://www.cancer.gov/ Search/SearchClinicalTrialsAdvanced.aspx, and where available, the corporate trial registries and databases of the manufacturers of the drugs. Where the underlying trial could unambiguously be identified in a registry or registries (by therapy protocols, inclusion criteria, patient numbers, location, commencement date, etc.), the earliest recorded date and place of registration was noted. For analysis, only reports of trials registered in a year prior to the earliest year of publication of results were classified as registered. Some registered trial reports did not meet this condition, as registration became a precondition of publication in many journals during the interval studied and post-hoc registration was allowed to satisfy this requirement. Sixteen of the 83 papers coded as unregistered appeared to have been registered in the same year as the first report, thus presumably after trial completion.

\section{Publication characteristics}

\section{Peer Review Status}

Each article was classified as peer reviewed or non-peer reviewed based on information found on the website of the journal where the article was published. A publication was considered peer reviewed if the website mentioned that the journal had a peer review process or if it was stated that the manuscripts were evaluated by at least one external expert in the field; otherwise, a publication was considered non-peer reviewed.

\section{Impact Factor}

For each article, impact factor for the journal in the year of publication was obtained from the Institute for Scientific Information [42].

\section{Main report}

Trial reports were classified as 1) first or main reports, if they were the earliest article in a given drug's publication sampling interval reporting results on a particular trial's primary efficacy outcome measure in the full patient population, or 2) subsidiary reports if an earlier publication in the sampling interval had disclosed results of the primary outcome measure. 


\section{Study design characteristics Comparison group}

The comparator for the primary outcome was classified as 1) placebo controlled, or 2) drug versus other treatment only. In drug versus other treatment comparisons involving two different oncology drugs of interest, the "test" drug was defined as the newer drug (most recent FDA approval date) and the older drug as the "comparator".

If a trial included multiple treatment arms, the placebo arm was treated as the comparator arm if present. If trials included multiple arms differing in dosage of the oncology drug of interest, the arm specifying the higher cumulative dosage was treated as the "test" arm whenever possible. If a publication reported results from multiple trials, the trial with the largest number of patients randomized was coded.

\section{Equivalency or Superiority trial}

Articles were classified as 1) equivalency/noninferiority trial if stated in the publication or 2) superiority trial.

\section{Type of primary outcome measures}

The preferred efficacy outcome measure recommended by the FDA for trials of antimalignancy therapies in this period was overall survival [43]. We classified the primary efficacy outcome reported in a publication as 1) overall survival or an equivalent measure (e.g. time to death) or 2) surrogate, if a surrogate measure was specified as the primary outcome. If no primary efficacy outcome was stated, or more than one "primary" outcome, the most emphasized efficacy outcome in the publication was designated the main outcome and treated as primary. In such cases we operationalized "most emphasized" according to word count in the results section, or if word count was about equal, the outcome discussed first. Identification of primary outcome measure was double-coded with high agreement (although kappa cannot be calculated because the number of possible outcome measures is indefinite).

\section{Blinding}

Blinding of treatment group allocation was classified on a scale of 0 to 3 based on the scheme of Chalmers et al. [44], with one point each for procedures assuring that patients were unaware of their allocation, that treating physicians and other patient care personnel were unaware, and that those evaluating the primary efficacy endpoint were unaware. If papers stated only that they were "double blind" without specifying procedures, they were classified with only one point. For analysis, we dichotomized blinding into "stringent" (score of 2 or 3 ) or "nonstringent" (score of 0 or 1$)$.

\section{Sample Size}

We recorded the total number of patients randomized to all treatments as described in the article, or total number enrolled if number randomized was not stated.

\section{Statistical Significance of Primary Outcome}

For each report, the results described for the primary efficacy outcome were categorised as 1) favorable if the result was statistically significant $(\mathrm{p}<0.05$ unless more stringent criteria were specified) and in the direction of the test drug being more efficacious, or 2) inconclusive if the result did not reach statistical significance, or 3) unfavorable if the result was statistically significant in the direction of the comparator treatment being more efficacious. If a paper explicitly stated that it was designed as a non-inferiority or equivalency study and no significant efficacy difference between the two comparison treatments was reported, the result was coded as favorable. For analysis, conclusions were dichotomised as favorable or not (combining inconclusive and unfavorable).

\section{Conclusion}

The overall conclusions reached in the published reports were categorised as 1) favorable, if the test drug was preferred to comparator, 2) equivalent if the test drug was described as about equal to comparator, or 3) unfavorable, if the comparator was preferred to the test drug. If an article did not clearly state that one of the treatments was better or if the comparison treatments had different but balanced advantages, the conclusion was coded as "test drug equivalent to comparator". If a study explicitly stated that it was designed as a non-inferiority or equivalency trial and the comparison treatments were said to be about equal, the conclusion was coded as favorable. For analysis, conclusions were dichotomized as favorable or not (combining equivalent and unfavorable).

\section{Sponsorship information \\ Funding source}

The funding source(s) of the published reports were categorized as 1) industry, 2) private non-profit, 3) government, 4) other, 5) no funding, 6) none disclosed.

\section{Financial ties}

Data about the financial ties of each author were extracted and coded for 1) whether or not there were ties of employment, research funding, consulting, stock ownership, or honoraria disclosed with the sponsor of the study (yes, no, or none disclosed), and 2) whether or not there were these same financial ties disclosed with any other company (yes, no, or none disclosed).

For analysis these categories were collapsed into two classes of sponsorship status, 1) sponsored by the test drug maker, if such a funding source for the trial was 
acknowledged explicitly, or where any author listed a relevant corporate address or employment, and 2) not commercially sponsored, if neither of the preceding categories applied. Supply of materials, or consulting or honoraria (etc.) on the part of authors did not in themselves qualify as sponsorship. Only one study was sponsored by the manufacturer of the comparator [45], and for analysis the paper was recoded as if the comparator (in fact an even newer drug) were the test drug.

\section{Analysis}

We report frequencies of the different characteristics of each article by registration status of the trial. Proportions of reports with favorable results or conclusions were first analyzed using univariate logistic regression to identify associations between independent variables and favorable results and conclusions. Although impact factor and sample size were continuous variables, they were modeled categorically by quartile because their effects were clearly non-linear. An exploration of interactions between trial and publication characteristics revealed no interactions except between trial sample size and journal impact factor (see Results, below).

To control for multiple variables simultaneously, we carried out multivariate logistic regression analysis and calculated odds ratios. These models included registration status as our primary predictor and all factors statistically associated either in the literature or in our univariate models with favorable results or conclusions. The natural $\log$ of sample size was treated as a numeric predictor. For our primary analysis, we conducted the multivariate regression on our full sample $(\mathrm{n}=137)$. We carried out pre-planned subgroup analyses of industry-sponsored studies only $(\mathrm{n}=109)$, and first or main reports only (i.e., one report per trial, $\mathrm{n}=115$ ). We also assessed sensitivity of our results to our definition of registration by repeating the primary multivariate analysis with both less stringent definitions of advance trial registration (any evidence of registration) and more stringent (evidence of registration in a year at least two years prior to publication). Data were analyzed with SAS software (version 9.1, SAS Institute, Cary, NC, USA).

\section{Results}

As designed this study required a sample of 116 included reports to achieve $80 \%$ power, assuming that $50 \%$ of reports from registered trials would describe favorable results and $75 \%$ from unregistered trials, and equal numbers of the two categories. Our actual full sample consisted of 137 trial reports meeting inclusion criteria (see Figure 1), and provided $85 \%$ power to distinguish the expected proportions. The sample characteristics are shown in Table 1. Thirty-nine percent (54/137) described trials registered prior to the year of the first publication.
The majority of both registered and unregistered trial reports were published in peer reviewed journals, did not describe stringent blinding, reported on a surrogate primary efficacy outcome measure, and were sponsored by the test drug maker. Fifty-eight percent (80/137) of the trial reports described results that were statistically significant in favor of the test drug for the primary efficacy outcome. Seventy-two percent $(98 / 137)$ had conclusions that favored the test drug.

Table 2 shows the results of univariate analysis of the full sample. Reports of trials with large sample sizes were significantly more likely to describe results and conclusions favoring the test drug, as were reports published in higher impact journals. Reports of trials sponsored by the maker of a test drug were significantly more likely to reach conclusions favoring the drug than non-sponsored trial reports. We found no statistically significant difference in results or conclusions between registered and non-registered trial reports. Published reports of registered trials were as likely to describe primary outcome results $(\mathrm{OR}=$ $1.77 ; 95 \% \mathrm{CI}=0.87$ to 3.61 ) and conclusions ( $\mathrm{OR}=1.99$; $95 \% \mathrm{CI}=0.89$ to 4.45 ) favoring the test drug compared to reports of non-registered trials.

In addition to registration status, we included industry sponsorship and sample size in our multivariate analysis because of their statistical significance in the univariate analysis. We also included stringency of blinding, outcome measure type, and comparator treatment type as these trial characteristics had been associated with direction of results in similar studies $[9,11,13,46]$. Journal impact factor was dropped from multivariate analysis because interaction testing found that its correlation with favorable results was accounted for by sample size. There was no interaction between stringency of blinding and surrogate outcome measure, or among any other variables. In the multivariate analysis (Table 3), reports of registered trials were again as likely to describe results favoring the test drug $(\mathrm{OR}=1.29 ; 95 \% \mathrm{CI}=0.54$ to 3.08$)$, and to reach favorable conclusions $(\mathrm{OR}=1.56 ; 95 \% \mathrm{CI}=$ 0.60 to 4.05 ). Reports of trials with surrogate outcome measures and large sample sizes (natural log) were significantly more likely to describe results favoring the test drug ( $p=0.027$ and $p<0.001$ respectively). Large sample size was a significant predictor of conclusions favoring the test drug $(\mathrm{p}=0.006)$ in the multivariate analysis.

For the subset of trial reports sponsored by the test drug makers $(n=109)$, results were essentially the same as for the full set, reports of registered trials being as likely to describe favorable results $(\mathrm{OR}=1.37 ; 95 \% \mathrm{CI}=0.54$ to $3.44)$. We also conducted a multivariate analysis for the subgroup of first or main reports $(n=115)$, including only one report per underlying trial (Table 4). As with the 
Table I: Characteristics of included articles by registration status $(n=137)$

\begin{tabular}{|c|c|c|}
\hline Characteristic & $\begin{array}{l}\text { Prior registration } \\
N=54\end{array}$ & $\begin{array}{l}\text { No Prior Registration } \\
\mathbf{N}=83\end{array}$ \\
\hline \multicolumn{3}{|l|}{ Publication characteristics } \\
\hline Peer reviewed & $53(98 \%)$ & $76(92 \%)$ \\
\hline \multicolumn{3}{|l|}{ Year of publication } \\
\hline $1996-2002$ & $0(0 \%)$ & $29(35 \%)$ \\
\hline $2003-2004$ & $12(22 \%)$ & 28 (34\%) \\
\hline $2005-2006$ & $14(26 \%)$ & $19(23 \%)$ \\
\hline $2007-2008$ & $28(52 \%)$ & $7(8 \%)$ \\
\hline Impact factor, median (interquartile range)* & $15.5(10.9-34.8)$ & $5.8(4.0-10.5)$ \\
\hline First or main report & $45(83 \%)$ & $70(84 \%)$ \\
\hline \multicolumn{3}{|l|}{ Study design characteristics } \\
\hline \multicolumn{3}{|l|}{ Comparison group } \\
\hline Placebo & $40(74 \%)$ & $32(39 \%)$ \\
\hline Active treatment only & $14(26 \%)$ & $51(62 \%)$ \\
\hline Non-inferiority trial - Yes & $2(4 \%)$ & $9(11 \%)$ \\
\hline Primary efficacy outcome = survival & $21(39 \%)$ & $9(11 \%)$ \\
\hline Stringent blinding ( $2 / 3$ points) & $16(30 \%)$ & $20(24 \%)$ \\
\hline Sample size, median (interquartile range) & $696(150-923)$ & $283(99-565)$ \\
\hline \multicolumn{3}{|l|}{ Statistical significance of primary outcome } \\
\hline Favorable to test drug & $36(67 \%)$ & $44(53 \%)$ \\
\hline Inconclusive & 18 (33\%) & 37 (45\%) \\
\hline Unfavorable & $0(\%)$ & $2(2 \%)$ \\
\hline \multicolumn{3}{|l|}{ Conclusion } \\
\hline Favorable to test drug & $43(80 \%)$ & $55(66 \%)$ \\
\hline About equal & $10(18 \%)$ & $22(27 \%)$ \\
\hline Unfavorable & I (2\%) & $6(7 \%)$ \\
\hline Sponsored by test drug maker & 47 (87\%) & $62(75 \%)$ \\
\hline
\end{tabular}

*Only 127 articles were published in journals with a corresponding impact factor. 


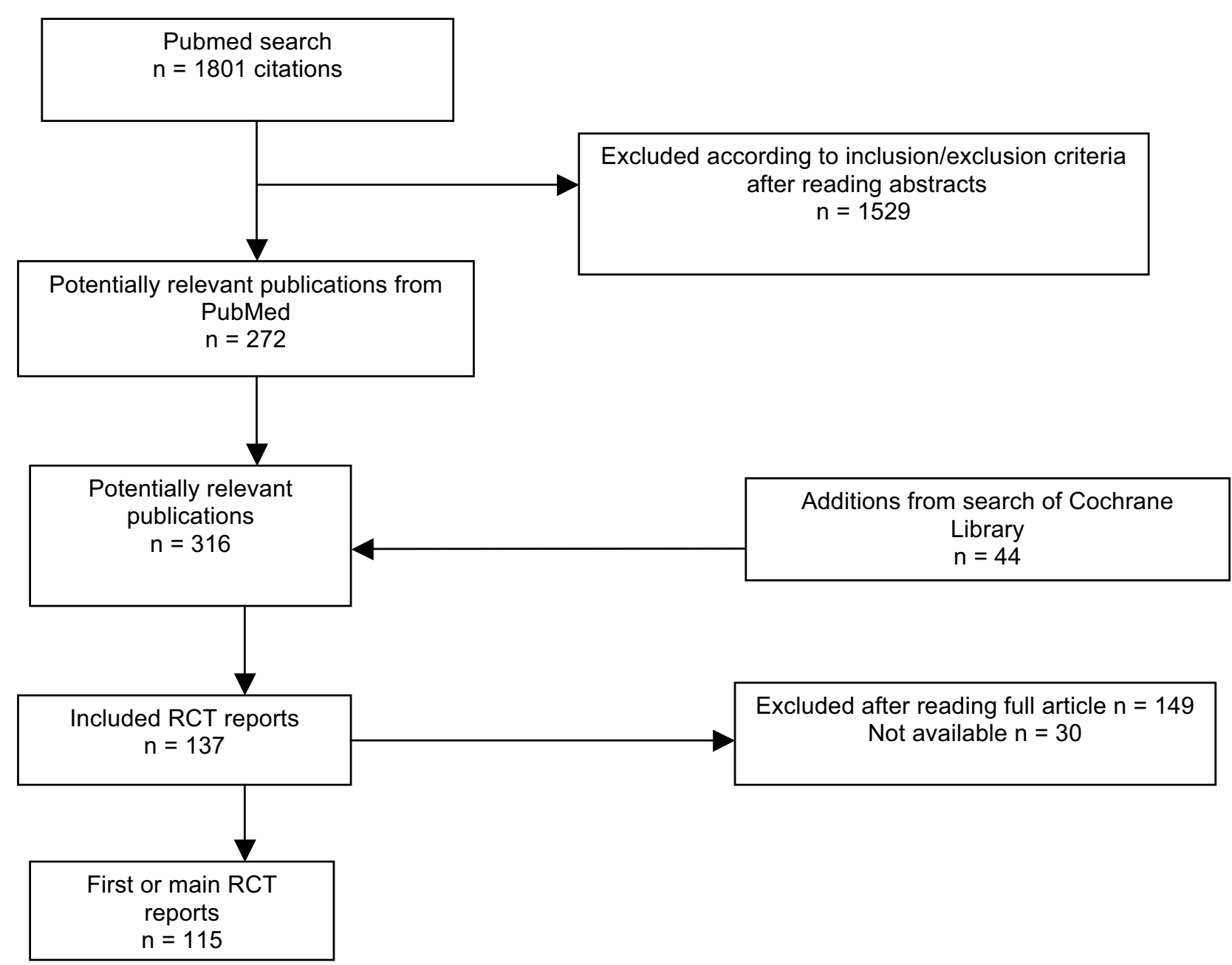

Figure I

Identification of trial reports for inclusion.

full set of publications, we did not find a statistically significant difference between registered and non-registered trials in the reporting of favorable results or conclusions. Published registered trials were as likely to report results favoring the test drug $(\mathrm{OR}=1.11 ; 95 \% \mathrm{CI}=0.44$ to 2.80$)$. Results only differed from the full sample multivariate analysis in that published trials with nonstringent blinding were significantly more likely to report results favoring the test drug ( $p=0.028)$, whereas surrogate outcome measure no longer reached significance as a predictor of results, indicating that this latter trial characteristic was over-represented among multiply-published trials.

A sensitivity analysis of our definition of prior registration yielded qualitatively similar results for the different definitions. The more stringent definition of prior registration (two years or more before first publication year) in our multivariate model indicated that reports of registered trials were at least as likely to describe favorable results (OR $=1.58 ; 95 \% \mathrm{CI}=0.65$ to 3.86 ) as compared with our basic definition. The less stringent definition (any evidence of registration) had an effect in the opposite direction (OR = $0.72 ; 95 \% \mathrm{CI}=0.30$ to 1.72 ), suggesting that the timing of trial registration may be a variable worthy of further study.

\section{Discussion}

We examined the association of trial registration and the results and conclusions of published randomized controlled trials of new oncology drugs. We found, contrary to expectations, that published reports of pre-registered trials were as likely to describe results favoring the test drug as non-registered trials, even when controlling for other 
Table 2: Association between characteristics of articles and statistically significant results or conclusions that favor the test drug: Univariate logistic regression $(n=137)$.

\begin{tabular}{|c|c|c|c|c|c|c|c|}
\hline \multirow[b]{2}{*}{ Characteristic } & \multirow[b]{2}{*}{ Category } & \multicolumn{3}{|c|}{ Results Favor Test Drug } & \multicolumn{3}{|c|}{ Conclusions Favor Test Drug } \\
\hline & & $\begin{array}{c}\text { Favorable } n / \text { Total } \\
n(\%)\end{array}$ & $\begin{array}{c}\text { OR } \\
(95 \% \mathrm{Cl})\end{array}$ & $P$ & $\begin{array}{c}\text { Favorable } n / \text { Total } \\
n(\%)\end{array}$ & $\begin{array}{c}\text { OR } \\
(95 \% \mathrm{Cl})\end{array}$ & $P$ \\
\hline \multirow{2}{*}{$\begin{array}{l}\text { Trial registration } \\
\text { before publication }\end{array}$} & No & $44 / 83(53)$ & 1.00 & & $55 / 83(66)$ & 1.00 & \\
\hline & Yes & $36 / 54(67)$ & $1.77(0.87-3.6 \mathrm{I})$ & 0.115 & $43 / 54(80)$ & $1.99(0.89-4.45)$ & 0.093 \\
\hline \multirow[t]{4}{*}{ Year of publication } & $1996-2002$ & $16 / 29(55)$ & 1.00 & & $21 / 29(72)$ & 1.00 & \\
\hline & $2003-2004$ & $27 / 40(68)$ & $1.69(0.63-4.53)$ & 0.299 & $30 / 40(75)$ & $1.14(0.39-3.38)$ & 0.809 \\
\hline & $2005-2006$ & $18 / 33(55)$ & $0.98(0.36-2.66)$ & 0.961 & $20 / 33(61)$ & $0.59(0.20-1.7 I)$ & 0.328 \\
\hline & $2007-2008$ & $19 / 35(54)$ & $0.97(0.36-2.59)$ & 0.943 & $27 / 35(77)$ & $1.29(0.4 \mathrm{I}-4.00)$ & 0.664 \\
\hline \multirow[t]{4}{*}{ Impact Factor* } & $\begin{array}{l}\text { Quartile I } \\
\text { (1.55-4.44) }\end{array}$ & $15 / 32(47)$ & 1.00 & & $19 / 32(59)$ & 1.00 & \\
\hline & $\begin{array}{l}\text { Quartile 2 } \\
(4.45-10.44)\end{array}$ & 19/33 (58) & $1.54(0.58-4.10)$ & 0.389 & $22 / 33(67)$ & $1.37(0.50-3.76)$ & 0.543 \\
\hline & $\begin{array}{l}\text { Quartile } 3 \\
\text { (10.45-17.15) }\end{array}$ & $20 / 35(57)$ & $1.51(0.58-3.96)$ & 0.402 & $26 / 35(74)$ & $1.98(0.70-5.57)$ & 0.197 \\
\hline & $\begin{array}{l}\text { Quartile 4 } \\
\text { (17.16-52.59) }\end{array}$ & $21 / 27(78)$ & $3.97(1.27-12.43)$ & 0.018 & 24/27 (89) & $5.47(1.36-22.01)$ & 0.017 \\
\hline \multirow[t]{2}{*}{ Blinding } & Stringent & $18 / 36(50)$ & 1.00 & & $25 / 36(69)$ & 1.00 & \\
\hline & Not stringent & $62 / 101(61)$ & $1.59(0.74-3.42)$ & 0.236 & 73/10I (72) & $1.15(0.50-2.64)$ & 0.747 \\
\hline \multirow[t]{4}{*}{ Sample Size } & Quartile I (6-I22) & 10/34 (29) & 1.00 & & $19 / 34(56)$ & 1.00 & \\
\hline & $\begin{array}{l}\text { Quartile } 2 \\
\text { (123-352) }\end{array}$ & $22 / 34(65)$ & $4.40(1.59-12.19)$ & 0.004 & 24/34 (7I) & $1.90(0.70-5.16)$ & 0.211 \\
\hline & $\begin{array}{l}\text { Quartile } 3 \\
\text { (353-772) }\end{array}$ & $22 / 35(63)$ & $4.06(1.48-11.12)$ & 0.006 & $26 / 35(74)$ & $2.28(0.83-6.30)$ & 0.112 \\
\hline & $\begin{array}{l}\text { Quartile 4 } \\
(773-8010)\end{array}$ & $26 / 34(76)$ & $7.8(2.64-23.03)$ & $<0.001$ & $29 / 34(85)$ & $4.58(1.43-14.69)$ & 0.011 \\
\hline \multirow{2}{*}{$\begin{array}{l}\text { Primary efficacy } \\
\text { outcome }\end{array}$} & Survival & $17 / 30(57)$ & 1.00 & & $21 / 30(70)$ & 1.00 & \\
\hline & Surrogate & 63/107 (59) & $1.10(0.48-2.48)$ & 0.828 & $77 / 107$ (72) & $1.10(0.45-2.67)$ & 0.833 \\
\hline \multirow[t]{2}{*}{ Comparison group } & Active comparator & $35 / 65(54)$ & 1.00 & & $45 / 65(69)$ & 1.00 & \\
\hline & Placebo & $45 / 72(63)$ & $0.70(0.35-1.39)$ & 0.306 & $53 / 72(74)$ & $0.81(0.38-1.70)$ & 0.571 \\
\hline \multirow[t]{2}{*}{ Sponsored } & No & $14 / 28(50)$ & 1.00 & & I5/28 (54) & 1.00 & \\
\hline & Yes & $66 / 109(61)$ & $1.54(0.67-3.54)$ & 0.314 & $83 / 109(76)$ & $2.77(1.17-6.56)$ & 0.021 \\
\hline
\end{tabular}

*Only 127 articles were published in journals with a corresponding impact factor. 
important predictors of statistical significance of results, including trial sample size, blinding, and industry sponsorship. Reports of registered trials in our sample actually appear more likely to favor new drugs (though not significantly); if so, one possible explanation is that trials expected to reflect favorably on new drugs were registered early.

Our findings argue for measures in addition to trial registration alone to ensure that statistically non-significant results are fully reported. Public access to statistically nonsignificant results is essential for conducting valid metaanalysis, advancing science, and enabling clinical decisions based on complete evidence [47-49]. Issues about the quality, assessment and presentation of non-peer- reviewed data in trial registers must be resolved, along with commercial concerns, without undue delay $[30,48]$. However, given the evidence that particular trial design features were associated with reported results, notwithstanding registration, our findings also suggest that results access through registries alone may never entirely eliminate the bias favoring new drugs in the clinical trial literature.

It might be argued that many registered "negative" trials of these recent oncology drugs have not yet been completed, or not yet submitted for publication, or that they have been rejected multiple times by journals. However, previous studies suggest that authors' decisions not to submit manuscripts, rather than journal rejection, account for the

Table 3: Association between characteristics of articles and statistically significant outcome or conclusions that favor the test drug: Multivariate logistic regression (full sample, $n=137$ )

\begin{tabular}{|c|c|c|c|c|c|c|c|}
\hline \multirow[b]{2}{*}{ Characteristic } & \multirow[b]{2}{*}{ Category } & \multicolumn{3}{|c|}{ Results Favor Test Drug } & \multicolumn{3}{|c|}{ Conclusions Favor Test Drug } \\
\hline & & $\begin{array}{c}\text { Favorable } \mathrm{n} / \text { Total } \\
\mathrm{n}(\%)\end{array}$ & $\begin{array}{c}\text { OR } \\
(95 \% \mathrm{Cl})\end{array}$ & $P$ & $\begin{array}{c}\text { Favorable } n / \text { Total } \\
n(\%)\end{array}$ & $\begin{array}{c}\text { OR } \\
(95 \% \mathrm{Cl})\end{array}$ & $P$ \\
\hline \multirow{2}{*}{$\begin{array}{l}\text { Trial registration } \\
\text { before publication }\end{array}$} & No & $44 / 83(53)$ & 1.00 & & $55 / 83(66)$ & 1.00 & \\
\hline & Yes & $36 / 54(67)$ & $1.29(0.54-3.08)$ & 0.566 & $43 / 54(80)$ & $1.56(0.60-4.05)$ & 0.363 \\
\hline \multirow[t]{2}{*}{ Blinding } & Stringent & $18 / 36(50)$ & 1.00 & & $25 / 36(69)$ & 1.00 & \\
\hline & Not stringent & $62 / 101(61)$ & $2.33(0.95-5.76)$ & 0.066 & 73/I0I (72) & $1.51(0.59-3.91)$ & 0.394 \\
\hline Sample Size & Natural log & - & $2.28(1.49-3.48)$ & $<0.001$ & - & $1.77(1.18-2.66)$ & 0.006 \\
\hline \multirow[t]{2}{*}{ Comparison group } & Active comparator & $35 / 65(54)$ & 1.00 & & $45 / 65(69)$ & 1.00 & \\
\hline & Placebo & $45 / 72(63)$ & $2.06(0.87-4.90)$ & 0.101 & $53 / 72(74)$ & $\mathrm{I} .47(0.59-3.63)$ & 0.410 \\
\hline \multirow{2}{*}{$\begin{array}{l}\text { Primary efficacy } \\
\text { outcome }\end{array}$} & Survival & $17 / 30(57)$ & 1.00 & & $21 / 30(70)$ & 1.00 & \\
\hline & Surrogate & 63/107 (59) & $3.42(1.15-10.14)$ & 0.027 & $77 / 107$ (72) & $3.04(0.95-9.68)$ & 0.061 \\
\hline \multirow[t]{2}{*}{ Sponsored } & No & $14 / 28(50)$ & 1.00 & & I5/28 (54) & 1.00 & \\
\hline & Yes & $66 / 109(61)$ & $1.01(0.37-2.67)$ & 0.999 & $83 / 109(76)$ & $2.01(0.76-5.3 I)$ & 0.157 \\
\hline
\end{tabular}


Table 4: Association between characteristics of trials and statistically significant outcome or conclusions favoring the test drug: Multivariate logistic regression (first/main reports only, $n=1$ I 5)

\begin{tabular}{|c|c|c|c|c|c|c|c|}
\hline \multirow[b]{2}{*}{ Characteristic } & \multirow[b]{2}{*}{ Category } & \multicolumn{3}{|c|}{ Results Favor Test Drug } & \multicolumn{3}{|c|}{ Conclusions Favor Test Drug } \\
\hline & & $\begin{array}{c}\text { Favorable } \mathbf{n} / \text { Total } \\
\mathbf{n}(\%)\end{array}$ & $\begin{array}{c}\text { OR } \\
(95 \% \mathrm{Cl})\end{array}$ & $\mathbf{P}$ & $\begin{array}{c}\text { Favorable } n / \text { Total } \\
n(\%)\end{array}$ & $\begin{array}{c}\text { OR } \\
(95 \% \mathrm{Cl})\end{array}$ & $\mathbf{P}$ \\
\hline \multirow{2}{*}{$\begin{array}{l}\text { Trial registration } \\
\text { before publication }\end{array}$} & No & $34 / 70(49)$ & 1.00 & & $46 / 70(66)$ & 1.00 & \\
\hline & Yes & $28 / 45(62)$ & I.II (0.44-2.80) & 0.832 & $34 / 45(76)$ & $1.10(0.41-2.98)$ & 0.854 \\
\hline \multirow[t]{2}{*}{ Blinding } & Stringent & $|3 / 3|(42)$ & 1.00 & & $20 / 3 \mid(65)$ & 1.00 & \\
\hline & Not stringent & $49 / 84(58)$ & $3.03(1.13-8.16)$ & 0.028 & $60 / 84(7 \mathrm{I})$ & $1.86(0.69-5.05)$ & 0.221 \\
\hline Sample size & Natural log & - & $2.20(1.40-3.45)$ & $<0.001$ & - & $1.65(1.09-2.5 I)$ & 0.019 \\
\hline \multirow[t]{2}{*}{ Comparison group } & Active comparator & $24 / 5$ I (47) & 1.00 & & $33 / 51(65)$ & 1.00 & \\
\hline & Placebo & $38 / 64(59)$ & $2.33(0.89-6.07)$ & 0.084 & $47 / 64(73)$ & $1.98(0.73-5.4 I)$ & 0.182 \\
\hline \multirow{2}{*}{$\begin{array}{l}\text { Primary efficacy } \\
\text { outcome }\end{array}$} & Survival & $14 / 25(56)$ & 1.00 & & $17 / 25(68)$ & 1.00 & \\
\hline & Surrogate & $48 / 90(53)$ & $2.76(0.82-9.28)$ & 0.102 & $63 / 90(70)$ & $2.86(0.80-10.18)$ & 0.105 \\
\hline \multirow[t]{2}{*}{ Sponsored } & No & 13/27 (48) & 1.00 & & $15 / 27(56)$ & 1.00 & \\
\hline & Yes & $49 / 88(56)$ & $1.02(0.37-2.84)$ & 0.965 & $65 / 88(74)$ & $1.94(0.7 \mathrm{I}-5.29)$ & 0.194 \\
\hline
\end{tabular}

majority of unpublished studies with statistically insignificant results $[2,3,50,51]$

Our study has several limitations. We focused on oncology drugs because they were most likely to include a sample of registered trials. Thus, our findings may not be generalizable to other classes of drugs. We were not able to examine trials of drugs that were never approved by the FDA as this list of drugs is not provided by the FDA. Our study design did not allow us to assess trials that were not published. In addition, to focus on trials of new therapies, we excluded studies published more than four years before or after the year of the oncology drug's approval in the 2000-2005 interval. Since trials with positive results are published significantly earlier than trials with negative results (4.3 yrs vs 6.5 yrs, respectively) [4], we may have failed to identify some studies yet to be published on the more recently approved drugs. However, these latter two limitations apply equally to prior registered and non-registered trials. We examined only the primary efficacy outcomes of the trials, and thus cannot determine whether trial registration might influence the reporting of secondary outcomes or safety outcomes. Lastly, trial registration has become increasingly common. Thus, our cross-sectional study offers only an early look at the effects of trial registration on results and conclusions in a particular sample of registered and unregistered trial reports. However, subsequent studies on the association of registration with reported results will be constrained to a before/after design and its attendant shortcomings. 


\section{Conclusions}

Trial registration alone, without a requirement for full reporting of research results, does not appear to reduce a bias toward results and conclusions favoring new drugs in the clinical trials literature. Our findings support the inclusion of full results reporting in trial registers, as well as measures to allow assessment of whether results have been completely reported. However, bias related to study design may remain. Possible policy solutions for design bias include advance review of trial protocols, compliance with existing reporting requirements, and adherence to best research practices.

\section{Competing interests}

The authors declare that they have no competing interests.

\section{Authors' contributions}

All three authors contributed to the conception and design of the study, acquisition of data, and analysis and interpretation of data. LB and NR drafted the manuscript, and all authors provided critical revision. All authors have had full access to all of the data in the study and take responsibility for the integrity of the data and the accuracy of the data analysis.

\section{Acknowledgements}

The project described was supported in part by grant KL2 RR024I 30 to Dr Lee from the National Center for Research Resources (NCRR), a component of the National Institutes of Health (NIH), and by grant DP0984694 to Dr Rasmussen from the Australian Research Council. The content is solely the responsibility of the authors and does not necessarily represent the official view of the NCRR or the National Institutes of Health or the Australian Research Council. The granting agencies had no role in the design and conduct of the study; collection, management, analysis and interpretation of the data; and preparation, submission, review or approval of the manuscript.

We thank Erika Campbell (UCSF) for administrative assistance and Peter Bacchetti, Ph.D., Chengshi Jin (both at UCSF) and Frances Garden (Sydney University) for help with statistical analyses. All were compensated for their work.

\section{References}

I. loannidis JP: Why most published research findings are false. PLoS Med 2005, 2:el 24.

2. Dickersin K, Chan S, Chalmers TC, Sacks HS, Smith H Jr: Publication bias and clinical trials. Control Clin Trials 1987, 8:343-353.

3. Dickersin K: The existence of publication bias and risk factors for its occurrence. Jama 1990, 263:1385-1389.

4. loannidis JP: Effect of the statistical significance of results on the time to completion and publication of randomized efficacy trials. Jama 1998, 279:28I-286.

5. Liss H: Publication bias in the pulmonary/allergy literature: effect of pharmaceutical company sponsorship. Isr Med Assoc | 2006, 8:45|-454.

6. Rasmussen $\mathrm{N}$ : The drug industry and clinical research in interwar America: three types of physician collaborator. Bull Hist Med 2005, 79:50-80.

7. Abaid LN, Grimes DA, Schulz KF: Reducing publication bias through trial registration. Obstet Gynecol 2007, I 09: | 434-I 437.

8. Colditz GA, Miller JN, Mosteller F: How study design affects outcomes in comparisons of therapy. I: Medical. Stat Med 1989, 8:44I-454.
9. loannidis JP, Cappelleri JC, Sacks HS, Lau J: The relationship between study design, results, and reporting of randomized clinical trials of HIV infection. Control Clin Trials 1997, I8:43|-444.

10. Schulz KF, Chalmers I, Hayes RJ, Altman DG: Empirical evidence of bias. Dimensions of methodological quality associated with estimates of treatment effects in controlled trials. Jama 1995, 273:408-4|2.

II. Bero L, Oostvogel F, Bacchetti P, Lee K: Factors associated with findings of published trials of drug-drug comparisons: why some statins appear more efficacious than others. PLOS Med 2007, 4:el84.

12. loannidis JP, Polycarpou A, Ntais C, Pavlidis N: Randomised trials comparing chemotherapy regimens for advanced non-small cell lung cancer: biases and evolution over time. Eur J Cancer 2003, 39:2278-2287.

13. Als-Nielsen B, Chen W, Gluud C, Kjaergard LL: Association of funding and conclusions in randomized drug trials: a reflection of treatment effect or adverse events? Jama 2003, 290:921-928.

14. Rochon PA, Gurwitz JH, Simms RW, Fortin PR, Felson DT, Minaker $\mathrm{KL}$, Chalmers TC: A study of manufacturer-supported trials of nonsteroidal anti-inflammatory drugs in the treatment of arthritis. Arch Intern Med 1994, 154:157-163.

15. Johansen HK, Gotzsche PC: Problems in the design and reporting of trials of antifungal agents encountered during metaanalysis. Jama 1999, 282:1752-1759.

16. Djulbegovic B, Lacevic M, Cantor A, Fields KK, Bennett CL, Adams $J R$, Kuderer NM, Lyman GH: The uncertainty principle and industry-sponsored research. Lancet 2000, 356:635-638.

17. Safer DJ: Design and reporting modifications in industry-sponsored comparative psychopharmacology trials. J Nerv Ment Dis 2002, 190:583-592.

18. Porta N, Bonet C, Cobo E: Discordance between reported intention-to-treat and per protocol analyses. J Clin Epidemiol 2007, 60:663-669.

19. Gravel J, Opatrny L, Shapiro S: The intention-to-treat approach in randomized controlled trials: are authors saying what they do and doing what they say? Clin Trials 2007, 4:350-356.

20. Chan AW, Krleza-Jeric K, Schmid I, Altman DG: Outcome reporting bias in randomized trials funded by the Canadian Institutes of Health Research. Cmaj 2004, I71:735-740.

21. Chan AW, Hrobjartsson A, Haahr MT, Gotzsche PC, Altman DG: Empirical evidence for selective reporting of outcomes in randomized trials: comparison of protocols to published articles. Jama 2004, 29 I:2457-2465.

22. Chan AW, Altman DG: Identifying outcome reporting bias in randomised trials on PubMed: review of publications and survey of authors. Bmj 2005, 330:753.

23. Lexchin J, Bero LA, Djulbegovic B, Clark O: Pharmaceutical industry sponsorship and research outcome and quality: systematic review. Bmj 2003, 326: II67-II 170 .

24. Bekelman JE, Li Y, Gross CP: Scope and impact of financial conflicts of interest in biomedical research: a systematic review. Jama 2003, 289:454-465.

25. Heres S, Davis J, Maino K, Jetzinger E, Kissling W, Leucht S: Why olanzapine beats risperidone, risperidone beats quetiapine, and quetiapine beats olanzapine: an exploratory analysis of head-to-head comparison studies of second-generation antipsychotics. Am J Psychiatry 2006, 163:185-194.

26. Fries JF, Krishnan E: Equipoise, design bias, and randomized controlled trials: the elusive ethics of new drug development. Arthritis Res Ther 2004, 6:R250-255.

27. Chalmers I: Underreporting research is scientific misconduct. Jama 1990, 263:| 1405-I408.

28. Rennie D: Fair conduct and fair reporting of clinical trials. Jama 1999, 282:1766-1768.

29. Krleza-Jeric K, Chan AW, Dickersin K, Sim I, Grimshaw J, Gluud C: Principles for international registration of protocol information and results from human trials of health related interventions: Ottawa statement (part I). Bmj 2005, 330:956-958.

30. Zarin DA, Ide NC, Tse T, Harlan WR, West JC, Lindberg DA: Issues in the registration of clinical trials. Jama 2007, 297:2I I 2-2I 20.

31 . Simes RJ: Publication bias: the case for an international registry of clinical trials. J Clin Oncol 1986, 4:|529-|54I. 
32. Egger M, Smith GD: Bias in location and selection of studies. Bmj 1998, 3 I 6:61-66.

33. Chalmers I: Unbiased, relevant, and reliable assessments in health care: important progress during the past century, but plenty of scope for doing better. Bmj 1998, 3 I 7:। I67-I I68.

34. Dickersin K, Rennie D: Registering clinical trials. Jama 2003, 290:516-523.

35. Levin LA, Palmer JG: Institutional review boards should require clinical trial registration. Arch Intern Med 2007, 167:1576-I580.

36. Doucet $M$, Sismondo S: Evaluating solutions to sponsorship bias. J Med Ethics 2008, 34:627-630.

37. Mathieu S, Boutron I, Moher D, Altman DG, Ravaud P: Comparison of registered and published primary outcomes in randomized controlled trials. Jama 2009, 302:977-984.

38. Ross JS, Mulvey GK, Hines EM, Nissen SE, Krumholz HM: Trial Publication after Registration in ClinicalTrials. Gov: A Cross-Sectional Analysis. PLoS Med 2009, 6:el000144.

39. Peppercorn J, Blood E, Winer E, Partridge A: Association between pharmaceutical involvement and outcomes in breast cancer clinical trials. Cancer 2007, 109:|239-1246.

40. Ramsey S, Scoggins J: Commentary: practicing on the tip of an information iceberg? Evidence of underpublication of registered clinical trials in oncology. Oncologist 2008, 13:925-929.

4I. Djulbegovic B, Kumar A, Soares HP, Hozo I, Bepler G, Clarke M, Bennett CL: Treatment success in cancer: new cancer treatment successes identified in phase 3 randomized controlled trials conducted by the National Cancer Institute-sponsored cooperative oncology groups, 1955 to 2006. Arch Intern Med 2008, I 68:632-642.

42. Institute for Scientific Information: Science Citation Index: Journal Citation Reports. Philadelphia, PA: Institute for Scientific Information; 2005.

43. Dagher R, Johnson J, Williams G, Keegan P, Pazdur R: Accelerated approval of oncology products: a decade of experience. J Natl Cancer Inst 2004, 96: I500-I509.

44. Chalmers I, Adams M, Dickersin K, Hetherington J, Tarnow-Mordi W, Meinert C, Tonascia S, Chalmers TC: A cohort study of summary reports of controlled trials. Jama 1990, 263:|40|-|405.

45. Kantarjian H, Pasquini R, Hamerschlak N, Rousselot P, Holowiecki J, Jootar S, Robak T, Khoroshko N, Masszi T, Skotnicki A, Hellmann A, Zaritsky A, Golenkov A, Radich J, Hughes T, Countouriotis A, Shah $\mathrm{N}$ : Dasatinib or high-dose imatinib for chronic-phase chronic myeloid leukemia after failure of first-line imatinib: a randomized phase 2 trial. Blood 2007, 109:5 I43-5I50.

46. Rochon PA, Binns MA, Litner JA, Litner GM, Fischbach MS, Eisenberg D, Kaptchuk TJ, Stason WB, Chalmers TC: Are randomized control trial outcomes influenced by the inclusion of a placebo group?: a systematic review of nonsteroidal antiinflammatory drug trials for arthritis treatment. J Clin Epidemiol 1999, 52: $113-122$

47. Ioannidis JP, Lau J: Improving safety reporting from randomised trials. Drug Saf 2002, 25:77-84.

48. Sim I, Detmer DE: Beyond trial registration: a global trial bank for clinical trial reporting. PLoS Med 2005, 2:e365.

49. Salvi VS: Research trials: registration, reporting and publication. J Postgrad Med 2005, 5 I:83-84.

50. Dickersin K, Min YI, Meinert CL: Factors influencing publication of research results. Follow-up of applications submitted to two institutional review boards. Jama 1992, 267:374-378.

5I. Lee KP, Boyd EA, Holroyd-Leduc JM, Bacchetti P, Bero LA: Predictors of publication: characteristics of submitted manuscripts associated with acceptance at major biomedical journals. Med J Aust 2006, 184:62I-626.
Publish with BioMed Central and every scientist can read your work free of charge

"BioMed Central will be the most significant development for disseminating the results of biomedical research in our lifetime. "

Sir Paul Nurse, Cancer Research UK

Your research papers will be:

- available free of charge to the entire biomedical community

- peer reviewed and published immediately upon acceptance

- cited in PubMed and archived on PubMed Central

- yours - you keep the copyright
BioMedcentral 